Z.S. Tan, MD, MPH

W.S. Harris, PhD

A.S. Beiser, PhD

R. Au, PhD

J.J. Himali, MS

S. Debette, MD

A. Pikula, MD

C. DeCarli, MD

P.A. Wolf, MD

R.S. Vasan, MD

S.J. Robins, MD

S. Seshadri, MD

Correspondence \& reprint requests to Dr. Tan: ztan@mednet.ucla.edu
Supplemental data at www.neurology.org

Supplemental Data

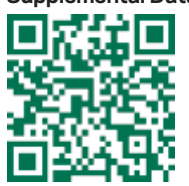

\title{
Red blood cell omega-3 fatty acid levels and markers of accelerated brain aging
}

\section{ABSTRACT}

Objective: Higher dietary intake and circulating levels of docosahexaenoic acid (DHA) and eicosapentaenoic acid (EPA) have been related to a reduced risk for dementia, but the pathways underlying this association remain unclear. We examined the cross-sectional relation of red blood cell (RBC) fatty acid levels to subclinical imaging and cognitive markers of dementia risk in a middleaged to elderly community-based cohort.

Methods: We related RBC DHA and EPA levels in dementia-free Framingham Study participants ( $n=1,575 ; 854$ women, age $67 \pm 9$ years) to performance on cognitive tests and to volumetric brain MRI, with serial adjustments for age, sex, and education (model A, primary model), additionally for APOE $\epsilon 4$ and plasma homocysteine (model B), and also for physical activity and body mass index (model C), or for traditional vascular risk factors (model D).

Results: Participants with RBC DHA levels in the lowest quartile (Q1) when compared to others (Q2-4) had lower total brain and greater white matter hyperintensity volumes (for model $A$ : $\beta \pm$ $\mathrm{SE}=-0.49 \pm 0.19 ; p=0.009$, and $0.12 \pm 0.06 ; p=0.049$, respectively) with persistence of the association with total brain volume in multivariable analyses. Participants with lower DHA and $\omega$-3 index (RBC DHA+EPA) levels (Q1 vs Q2-4) also had lower scores on tests of visual memory $(\beta \pm \mathrm{SE}=-0.47 \pm 0.18 ; p=0.008)$, executive function $(\beta \pm \mathrm{SE}=-0.07 \pm 0.03 ; p=0.004)$, and abstract thinking $(\beta \pm \mathrm{SE}=-0.52 \pm 0.18 ; p=0.004)$ in model $\mathrm{A}$, the results remaining significant in all models.

Conclusion: Lower RBC DHA levels are associated with smaller brain volumes and a "vascular" pattern of cognitive impairment even in persons free of clinical dementia. Neurology ${ }^{\circledR} 2012 ; 78: 658-664$

\section{GLOSSARY}

$\mathbf{A D}=$ Alzheimer disease; $\mathbf{B M I}$ = body mass index; $\mathbf{D H A}=$ docosahexaenoic acid; $\mathbf{D M}=$ diabetes mellitus; $\mathbf{E P A}=$ eicosapentaenoic acid; $\mathbf{L M}-\mathbf{d}$ = delayed recall components of the Logical Memory test; $\mathbf{N P}=$ neuropsychological; $\mathbf{P A I}=$ physical activity index; PUFA = polyunsaturated fatty acid; $\mathbf{R B C}=$ red blood cell; $\mathbf{S B P}=$ systolic blood pressure; $\mathbf{S C I}$ = silent cerebral infarct; $\mathbf{S I M}=$ Similarities test; $\mathbf{T C B V}=$ total cerebral brain volume; $\mathbf{T H V}=$ temporal horn volume; VR-d = delayed recall component of the Visual Reproductions test; WMHV = white matter hyperintensity volume.

Higher fish intake has been associated with a reduced risk of cardiovascular mortality ${ }^{1,2}$ and stroke. ${ }^{3}$ While several large epidemiologic studies, ${ }^{4,5}$ have shown an association between the estimated intake of fatty fish and a lower risk for dementia, some other investigations have failed to confirm such a protective association. ${ }^{6}$ One possible reason for these inconsistent results is the limited ability of dietary recall surveys and food frequency questionnaires to quantify blood levels of fatty acids. ${ }^{7,8}$

In the Framingham original cohort, participants in the top quartile of plasma phosphatidylcholine docosahexaenoic acid (DHA) levels had 37\% and 47\% lower risks of Alzheimer disease (AD) and all-cause dementia, respectively. ${ }^{9}$ Red blood cell (RBC) fatty acid composition re-

From the Department of Medicine, Division of Geriatric Medicine, and the Easton Center for Alzheimer's Disease Research (Z.S.T.), David Geffen School of Medicine at the University of California at Los Angeles, Los Angeles; Cardiovascular Health Research Center (W.S.H.), Sanford Research, University of South Dakota, Sioux Falls; Department of Biostatistics (A.S.B., J.J.H.), Boston University School of Public Health, Boston; Departments of Neurology (A.S.B., J.J.H., S.S., R.A., S.D., A.P., P.A.W.) and Medicine (RSV, SR), Boston University School of Medicine, Boston, MA; Department of Neurology (C.D.), University of California-Davis, Davis; and the Framingham Heart Study (Z.S.T., A.S.B., R.A., P.A.W., R.S.V., S.J.R., S.S.), Framingham, MA.

Study funding: Supported by the Framingham Heart Study's National Heart, Lung, and Blood Institute contract (N01-HC-25195) and by grants from the NHLBI: R01 HL089590 and National Institute on Aging (R01 AG16495, AG 033040, AG08122, AG033193, and AG031287).

Disclosure: Author disclosures are provided at the end of the article. 
flects dietary fatty acid intake averaged over the RBC lifespan of up to 120 days, whereas plasma concentrations reflect intake over only the last few days. ${ }^{10}$ Further RBC membrane omega-3 fatty acid composition is more biologically stable than plasma concentrations, ${ }^{11}$ and has been shown to be highly correlated with omega-3 fatty acid concentrations in tissues such as the heart. ${ }^{12}$ However, to our knowledge, no prior study has related RBC fatty acid composition to subclinical markers of future dementia.

The middle-aged Framingham Offspring cohort has been evaluated for subclinical markers of risk for dementia by brain MRI and cognitive (neuropsychological $[\mathrm{NP}]$ ) tests. We related RBC omega-3 fatty acid levels to recognized MRI and cognitive markers of subclinical $\mathrm{AD}$ and vascular pathology and of risk for dementia in a large, community-based sample.

METHODS Study sample. Framingham Offspring Study participants who attended offspring core examination 8 (between March 2005 and January 2008) had blood drawn for measurement of RBC fatty acid composition and were also invited to undergo brain MRI studies and a NP assessment at a subsequent call-back examination, typically about 3 months after the core Framingham Heart Study examination. Of 3,021 participants who attended the eighth Offspring examination, 2,900 had RBC fatty acid measurements, and of these, 1,664 had NP data. Persons with a contraindication to brain MRI (such as claustrophobia or a pacemaker) were excluded. A total of 36 participants were excluded for neurologic conditions that could affect MRI measurement (e.g., multiple sclerosis, brain tumor), 47 for prevalent stroke, and 6 for prevalent dementia, yielding our study sample of $\mathrm{n}=1,575$ ( 854 women, mean age $67 \pm 9$ years). The MRI and NP testing were performed on the same day for most participants, and in all cases, by evaluators blind to the RBC fatty acid data.

Standard protocol approvals, registrations, and participant consents. Data were obtained under a protocol approved by the Human Subjects Institutional Review Board of the Boston University School of Medicine. Written informed consent was obtained from all participants.

Brain MRI study. Image acquisition. The methods followed for brain MRI have previously been described. ${ }^{13,14}$ MRI measures assessed consisted of total cerebral brain volume (TCBV), temporal horn volume (THV, inversely related to hippocampal volume), white matter hyperintensity volume (WMHV), and silent cerebral infarcts (SCI). Brain volume was determined by manual outlining in coronal images of the intracranial vault above the tentorium to determine the total cranial volume as a function of head size. Once the skull and other nonbrain tissues were removed from the image, mathematical modeling was performed to determine total parenchymal brain volume above the tentorium (cerebral). Hippocampal volume was estimated using THV; since the medial wall of the temporal horn is comprised of the hippocampus, atrophy of the hippocampus will result in enlargement of the THV. Both TCBV and THV were calculated as ratios over total intracranial volume. WMHV was measured according to previously published methods ${ }^{15}$ and expressed as a proportion of total intracranial volume, and log-transformed to normalize its distribution. The presence or absence of an SCI was determined manually by the operator, based on the size $(\geq 3$ $\mathrm{mm})$, location, and imaging characteristics of the lesion. MRI infarcts were classified as SCIs if the person had not had a clinically documented stroke at any time before MRI. The inter-rater reliability for these measures has been previously reported. ${ }^{16}$

Cognitive evaluation. The NP battery has been previously described. ${ }^{17}$ From this battery we selected 3 tests that represent cognitive domains previously associated with an increased risk of developing $\mathrm{AD}$ in the original cohort ${ }^{18}$ : the delayed recall components of the Logical Memory test (LM-d) assessing verbal memory, the delayed recall component of the Visual Reproductions test (VR-d) assessing visuospatial memory, and the Similarities test (SIM) which measures abstract reasoning skills, and a fourth test (Trails B) which is a marker of attention and executive function and has been associated with vascular brain injury. Poorer performance on VR-d and SIM tests has also been associated with presence of vascular risk factors. ${ }^{19}$

RBC fatty acid analysis. RBCs were isolated from blood drawn after a 10- to 12 -hour fast and frozen at $-80^{\circ} \mathrm{C}$ immediately after collection. RBC fatty acid composition was analyzed according to the HS-Omega- 3 index ${ }^{\circledR}$ methodology. ${ }^{20}$ Briefly, fatty acid methyl esters were generated from RBCs by acid transesterification with boron trifluoride and analyzed by gas chromatography using a GC2010 Gas Chromatograph (Shimadzu Corporation, Columbia, MD) equipped with a SP2560, 100-m column (Supelco, Bellefonte, PA). Fatty acids were identified by comparison with a standard mixture of fatty acids characteristic of RBCs. After response factor correction, results were calculated as a percentage of total identified fatty acids (as membrane fatty acid composition is typically reported). From these values we derived the omega- 3 index (RBC EPA and DHA expressed as weight percentage of total fatty acids). ${ }^{21}$ In our study population, on average DHA consisted of $87.4 \%$ of the sum DHA+EPA. The interassay coefficient of variation was $4.9 \%$ for both EPA and DHA.

Statistical analyses. Although multiple RBC fatty acids were measured, we made an a priori decision to examine the associations of only RBC DHA and omega-3 index (independent variables) to the phenotypes described above (dependent variables) since these fatty acids had been previously associated with the risk of dementia. We used linear regression for the continuous phenotypes and logistic regression for the dichotomous phenotypes. We constructed multivariable regression models initially adjusting for age, sex, time interval between date of blood draw for measurement of fatty acids and date of brain MRI, and education (for cognitive measures) (model A). Of note, we adjusted for the time interval between the baseline examination when blood was drawn for RBC omega-3 fatty acid measurement and the date of MRI/NP testing since values of the covariates were more likely to have changed during this time interval if the interval was longer. For each phenotype, we first examined the linear effect of a 1 SD unit increase in RBC DHA, and also explored threshold models comparing the lowest quartiles of RBC DHA (Q1) to the combined group of the upper 3 quartiles (Q2-4). We then added additional covariates in sequential models; we adjusted for APOE $\epsilon 4$ allele status and homocysteine levels (model B), then also for body mass index (BMI) and physical 


\begin{tabular}{|c|c|c|}
\hline Table 1 & \multicolumn{2}{|c|}{$\begin{array}{l}\text { Characteristics of participants at } \\
\text { examination } 8 \text { (2005-2008) }\end{array}$} \\
\hline \multicolumn{2}{|c|}{ Characteristic } & Value \\
\hline \multicolumn{2}{|l|}{ No. } & 1,575 \\
\hline \multicolumn{2}{|c|}{ Age, $y$, mean $\pm S D$} & $67 \pm 9$ \\
\hline \multicolumn{2}{|c|}{ Women, \% } & 54 \\
\hline \multicolumn{2}{|c|}{ High school degree, $\%^{a}$} & 26 \\
\hline \multicolumn{2}{|c|}{ Current cigarette smoking, $\%$} & 8 \\
\hline \multicolumn{2}{|c|}{$\begin{array}{l}\text { Systolic blood pressure, } \mathrm{mm} \mathrm{Hg} \\
\text { mean } \pm \text { SD }\end{array}$} & $128 \pm 17$ \\
\hline \multicolumn{2}{|c|}{ APOE $4, \%$} & 23 \\
\hline \multicolumn{2}{|c|}{$\begin{array}{l}\text { Log plasma homocysteine, } \mu \mathrm{mol} / \mathrm{L}, \\
\text { mean } \pm \mathrm{SD}\end{array}$} & $2.05 \pm 0.29$ \\
\hline \multicolumn{2}{|c|}{ Total cholesterol, $\mathrm{mg} / \mathrm{dL}$, mean $\pm \mathrm{SD}$} & $186 \pm 37$ \\
\hline \multicolumn{2}{|c|}{ Prevalent CVD, \% } & 12 \\
\hline \multicolumn{2}{|c|}{ Diabetes, \% } & 13 \\
\hline \multicolumn{2}{|c|}{ Atrial fibrillation, \% } & 5 \\
\hline \multicolumn{2}{|c|}{ Physical activity index, mean \pm SD } & $35 \pm 6$ \\
\hline \multicolumn{2}{|c|}{ Body mass index, mean $\pm S D$} & $28 \pm 5$ \\
\hline \multicolumn{2}{|c|}{ Time interval exam 8 to MRI, $y$, mean \pm SD } & $0.29 \pm 0.82$ \\
\hline \multicolumn{2}{|c|}{$\begin{array}{l}\text { Time interval exam } 8 \text { to cognitive exam, } \\
y, \text { mean } \pm \text { SD }\end{array}$} & $0.29 \pm 0.84$ \\
\hline \multicolumn{3}{|c|}{$\begin{array}{l}\text { RBC omega-3 PUFA content (\% of total } \\
\text { RBC fatty acids) }\end{array}$} \\
\hline \multicolumn{3}{|l|}{ DHA } \\
\hline \multicolumn{2}{|c|}{ 25th percentile } & 3.9 \\
\hline \multicolumn{2}{|c|}{ Median } & 4.7 \\
\hline \multicolumn{2}{|c|}{ 75th percentile } & 5.7 \\
\hline \multicolumn{3}{|c|}{ DHA + EPA (omega-3 index) } \\
\hline \multicolumn{2}{|c|}{ 25th percentile } & 4.4 \\
\hline \multicolumn{2}{|c|}{ Median } & 5.3 \\
\hline \multicolumn{2}{|c|}{ 75th percentile } & 6.5 \\
\hline
\end{tabular}

Abbreviations: CVD = cerebrovascular disease; $\mathrm{DHA}=$ docosahexaenoic acid; EPA = eicosapentaenoic acid; PUFA = polyunsaturated fatty acid; RBC = red blood cell.

a High school education or above.

activity index (PAI) ${ }^{22}$ (model C), or for all traditional vascular risk factors diabetes mellitus (DM), systolic blood pressure (SBP), cigarette smoking, atrial fibrillation, prevalent cerebrovascular disease, and total cholesterol (model D). We chose model A as the primary analysis since we wished to screen for a possible association with lower tissue omega-3 fatty acid levels regardless of whether or not this association was partly mediated through vascular risk factors. ${ }^{23}$ All analyses were performed using SAS version 9.2 (SAS Institute Inc, Cary, NC).

RESULTS Results are illustrated in tables e- 1 and e-2 on the Neurology ${ }^{\circledR}$ Web site at www.neurology.org. The baseline characteristics of the participants are presented in table 1 , and characteristics stratified by baseline DHA and EPA levels are presented in table e-1.

Association of RBC DHA with MRI measures of brain aging. Results are presented in table 2. RBC DHA was not linearly related to any of the brain MRI mea- sures examined. However, there was a threshold effect, and comparing persons with RBC DHA levels in the lowest quartile to persons with a level in higher quartiles (Q2-4) using model A we observed a significantly lower TCBV. Additional adjustment for $A P O E \epsilon 4$ and homocysteine (model $\mathrm{B}$ ), BMI and PAI (model C), and for the factors associated with an increased risk of stroke, including cigarette smoking, which was more prevalent in Q1 than in Q2-4, did not attenuate this association (model D). Participants with Q1 of RBC DHA $(<3.9 \%)$ had greater WMHV compared to participants in Q2-4 $(>3.9 \%)$ in model A; this association was borderline significant in models $\mathrm{B}$ and $\mathrm{C}$ but became statistically nonsignificant after adjusting for vascular risk factors (models D). No statistically significant associations were observed between RBC DHA and THV or SCI in any of the models. Analyses using the omega-3 index yielded similar results: there was no linear relation to any of the brain MRI measures but low values (Q1 vs Q2-4; below vs above 4.4\%) were inversely associated with TCBV in all 4 models and positively associated with WMHV in model A. Similar to findings for DHA levels alone, there were no statistically significant associations observed between omega-3 index and THV or SCI.

Association of RBC DHA with cognitive measures of brain aging. Results are presented in table 3. RBC DHA levels showed a continuous positive association with performance in tests of visual memory (VR-d), executive function (Trails B), and abstract thinking (SIM), respectively. Similar results were found with omega-3 index and NP test performance. There were no statistically significant relationships between RBC DHA or omega- 3 index and performance on verbal memory (LM-d). Adjusting for covariates in all models did not alter the association between abstract thinking and DHA or omega-3 index, while the observations for visual memory and executive function were diminished in model D. Participants in the lowest quartile (Q1) of DHA levels performed more poorly on tests of visual memory, executive function, and abstract thinking compared to participants in the higher quartiles (Q2-4). This relationship remained significant in all 4 models. In contrast, we found no relationship between verbal memory and either DHA or omega-3 index in any of the models.

DISCUSSION In this cohort study, we found that lower levels of RBC DHA and EPA in late middle age were associated with markers of accelerated structural and cognitive aging. The MRI finding of lower brain volume represents a change equivalent to approximately 2 years of structural brain aging. These findings extend the observations of the Cardiovascu- 
Table 2 Association of RBC membrane levels of the omega-3 fatty acids, DHA, and EPA (as a percent of total RBC fatty acids) with MRI measures of brain aging ${ }^{\text {a }}$

\begin{tabular}{|c|c|c|c|c|}
\hline MRI measure & $\begin{array}{l}\text { Total cerebral brain } \\
\text { volume (percent) }\end{array}$ & $\begin{array}{l}\text { White matter } \\
\text { hyperintensity } \\
\text { volume (logWMHV) }\end{array}$ & $\begin{array}{l}\text { Temporal horn } \\
\text { volume } \\
\text { (logTHV) }\end{array}$ & $\begin{array}{l}\text { Silent cerebral } \\
\text { infarct (presence of } \\
\text { one or more) }\end{array}$ \\
\hline Mean + SD & $78.98 \pm 3.85$ & $0.18 \pm 0.33$ & $0.07 \pm 0.06$ & $14 \%$ \\
\hline Results & $\beta \pm \mathrm{SE}$ & $\beta \pm \mathrm{SE}$ & $\beta \pm \mathrm{SE}$ & OR $(95 \% \mathrm{Cl})$ \\
\hline \multicolumn{5}{|c|}{ RBC DHA (per SD unit) } \\
\hline \multirow[t]{2}{*}{ Model A } & $0.13 \pm 0.08$ & $-0.05 \pm 0.03$ & $-0.01 \pm 0.02$ & $1.00(0.85-1.18)$ \\
\hline & $p=0.127$ & $p=0.061$ & $p=0.746$ & $p=0.984$ \\
\hline \multirow[t]{2}{*}{ Model B } & $0.12 \pm 0.09$ & $-0.05 \pm 0.03$ & $-0.01 \pm 0.02$ & $1.02(0.87-1.20)$ \\
\hline & $p=0.148$ & $p=0.051$ & $p=0.794$ & $p=0.789$ \\
\hline \multirow[t]{2}{*}{ Model C } & $0.11 \pm 0.09$ & $-0.06 \pm 0.03$ & $-0.00 \pm 0.02$ & $1.01(0.86-1.18)$ \\
\hline & $p=0.211$ & $p=0.045^{b}$ & $p=0.884$ & $p=0.943$ \\
\hline \multirow[t]{2}{*}{ Model D } & $0.10 \pm 0.09$ & $-0.05 \pm 0.03$ & $-0.00 \pm 0.02$ & $1.01(0.86-1.20)$ \\
\hline & $p=0.271$ & $p=0.086$ & $p=0.995$ & $p=0.881$ \\
\hline \multicolumn{5}{|c|}{ RBC DHA (Q1 vs Q2-4) } \\
\hline \multirow{2}{*}{ Model A } & $-0.49 \pm 0.19$ & $0.12 \pm 0.06$ & $0.05 \pm 0.05$ & 0.99 (0.68-1.44) \\
\hline & $p=0.009^{b}$ & $p=0.049^{b}$ & $p=0.348$ & $p=0.959$ \\
\hline \multirow[t]{2}{*}{ Model B } & $-0.49 \pm 0.19$ & $0.12 \pm 0.06$ & $0.04 \pm 0.05$ & $0.97(0.67-1.40)$ \\
\hline & $p=0.011^{b}$ & $p=0.053$ & $p=0.421$ & $p=0.852$ \\
\hline \multirow[t]{2}{*}{ Model C } & $-0.48 \pm 0.19$ & $0.12 \pm 0.06$ & $0.04 \pm 0.04$ & $0.98(0.68-1.43)$ \\
\hline & $p=0.013^{b}$ & $p=0.057$ & $p=0.396$ & $p=0.923$ \\
\hline \multirow[t]{2}{*}{ Model D } & $-0.45 \pm 0.19$ & $0.10 \pm 0.06$ & $0.03 \pm 0.05$ & $0.96(0.66-1.40)$ \\
\hline & $p=0.019^{b}$ & $p=0.102$ & $p=0.595$ & $p=0.836$ \\
\hline \multicolumn{5}{|c|}{ RBC DHA + EPA (per SD unit) } \\
\hline \multirow[t]{2}{*}{ Model A } & $0.12 \pm 0.08$ & $-0.05 \pm 0.03$ & $-0.01 \pm 0.02$ & $1.01(0.86-1.18)$ \\
\hline & $p=0.145$ & $p=0.097$ & $p=0.800$ & $p=0.920$ \\
\hline \multirow[t]{2}{*}{ Model B } & $0.12 \pm 0.09$ & $-0.05 \pm 0.03$ & $-0.004 \pm 0.02$ & $1.03(0.88-1.21)$ \\
\hline & $p=0.163$ & $p=0.077$ & $p=0.849$ & $p=0.723$ \\
\hline \multirow[t]{2}{*}{ Model C } & $0.10 \pm 0.09$ & $-0.05 \pm 0.03$ & $-0.001 \pm 0.02$ & $1.01(0.86-1.19)$ \\
\hline & $p=0.243$ & $p=0.066$ & $p=0.948$ & $p=0.888$ \\
\hline \multirow[t]{2}{*}{ Model D } & $0.09 \pm 0.09$ & $-0.05 \pm 0.03$ & $0.001 \pm 0.02$ & $1.02(0.87-1.21)$ \\
\hline & $p=0.283$ & $p=0.111$ & $p=0.969$ & $p=0.798$ \\
\hline \multicolumn{5}{|c|}{ RBC DHA + EPA (Q1 vs Q2-4) } \\
\hline \multirow[t]{2}{*}{ Model A } & $-0.53 \pm 0.18$ & $0.12 \pm 0.06$ & $0.03 \pm 0.05$ & $1.01(0.70-1.46)$ \\
\hline & $p=0.005^{b}$ & $p=0.044^{b}$ & $p=0.540$ & $p=0.957$ \\
\hline \multirow[t]{2}{*}{ Model B } & $-0.54 \pm 0.19$ & $0.12 \pm 0.06$ & $0.02 \pm 0.05$ & $1.00(0.69-1.45)$ \\
\hline & $p=0.005^{b}$ & $p=0.062$ & $p=0.621$ & $p=0.998$ \\
\hline \multirow[t]{2}{*}{ Model C } & $-0.51 \pm 0.19$ & $0.12 \pm 0.06$ & $0.03 \pm 0.05$ & $1.02(0.71-1.48)$ \\
\hline & $p=0.008^{b}$ & $p=0.064$ & $p=0.611$ & $p=0.910$ \\
\hline \multirow[t]{2}{*}{ Model D } & $-0.49 \pm 0.19$ & $0.11 \pm 0.06$ & $0.01 \pm 0.05$ & (0.68-1.45) \\
\hline & $p=0.011^{b}$ & $p=0.097$ & $p=0.817$ & $p=0.980$ \\
\hline
\end{tabular}

Abbreviations: $\mathrm{Cl}$ = confidence interval; $\mathrm{DHA}=$ docosahexaenoic acid; $\mathrm{EPA}=$ eicosapentaenoic acid; OR = odds ratio; $\mathrm{RBC}=$ red blood cell.

a Model A: adjusted for age, sex, and time interval. Model B: model A with additional adjustment for APOE4 and homocysteine. Model C: model B with additional adjustment for physical activity index and body mass index. Model D: model B with additional adjustment for diabetes mellitus, systolic blood pressure, smoking, atrial fibrillation, prevalent cardiovascular disease, and total cholesterol. Higher white matter hyperintensity and temporal horn volumes and lower total brain volume are correlated with the presence of vascular risk factors.

b Significant. 
Table 3 Association of RBC membrane levels of the omega-3 fatty acids, DHA, and EPA (as a percent of total RBC fatty acids) with cognitive performance

\begin{tabular}{|c|c|c|c|c|}
\hline & $\begin{array}{l}\text { Logical } \\
\text { Memory- } \\
\text { delayed }\end{array}$ & $\begin{array}{l}\text { Visual } \\
\text { Reproductions- } \\
\text { delayed }\end{array}$ & $\begin{array}{l}\text { Trails B } \\
\text { (logTrB) }\end{array}$ & Similarities \\
\hline Mean + SD & $11.31 \pm 3.90$ & $8.13 \pm 3.40$ & $1.63 \pm 1.30$ & $17.12 \pm 3.55$ \\
\hline Results & $\beta \pm \mathrm{SE}$ & $\beta \pm \mathrm{SE}$ & $\beta \pm \mathrm{SE}$ & $\beta \pm \mathrm{SE}$ \\
\hline \multicolumn{5}{|l|}{$\begin{array}{l}\text { RBC DHA } \\
\text { (per SD unit) }\end{array}$} \\
\hline \multirow[t]{2}{*}{ Model A } & $0.11 \pm 0.09$ & $0.17 \pm 0.08$ & $0.03 \pm 0.01$ & $0.26 \pm 0.08$ \\
\hline & $p=0.242$ & $p=0.026^{b}$ & $p=0.025^{b}$ & $p=0.001^{b}$ \\
\hline \multirow[t]{2}{*}{ Model B } & $0.12 \pm 0.10$ & $0.18 \pm 0.08$ & $0.02 \pm 0.01$ & $0.26 \pm 0.08$ \\
\hline & $p=0.232$ & $p=0.026^{b}$ & $p=0.038^{b}$ & $p=0.002^{b}$ \\
\hline \multirow[t]{2}{*}{ Model C } & $0.13 \pm 0.10$ & $0.18 \pm 0.08$ & $0.02 \pm 0.01$ & $0.25 \pm 0.08$ \\
\hline & $p=0.179$ & $p=0.024^{b}$ & $p=0.046^{b}$ & $p=0.002^{b}$ \\
\hline Model D & $0.11 \pm 0.10$ & $0.14 \pm 0.08$ & $0.02 \pm 0.01$ & $0.27 \pm 0.08$ \\
\hline $\begin{array}{l}\text { RBC DHA } \\
\text { (Q1 vs Q2-4) }\end{array}$ & $p=0.258$ & $p=0.079$ & $p=0.108$ & $p=0.001^{b}$ \\
\hline \multirow[t]{2}{*}{ Model A } & $-0.18 \pm 0.22$ & $-0.47 \pm 0.18$ & $-0.07 \pm 0.03$ & $-0.52 \pm 0.18$ \\
\hline & $p=0.394$ & $p=0.008^{b}$ & $p=0.004^{b}$ & $p=0.004^{b}$ \\
\hline \multirow[t]{2}{*}{ Model B } & $-0.21 \pm 0.22$ & $-0.44 \pm 0.18$ & $-0.08 \pm 0.03$ & $-0.52 \pm 0.19$ \\
\hline & $p=0.334$ & $p=0.014^{b}$ & $p=0.005^{b}$ & $p=0.006^{b}$ \\
\hline \multirow[t]{2}{*}{ Model C } & $-0.22 \pm 0.22$ & $-0.44 \pm 0.18$ & $-0.07 \pm 0.03$ & $-0.51 \pm 0.19$ \\
\hline & $p=0.324$ & $p=0.015^{b}$ & $p=0.006^{b}$ & $p=0.007^{b}$ \\
\hline Model D & $-0.22 \pm 0.22$ & $-0.37 \pm 0.18$ & $-0.06 \pm 0.03$ & $-0.52 \pm 0.19$ \\
\hline $\begin{array}{l}\text { RBC DHA + EPA } \\
\text { (per SD Unit) }\end{array}$ & $p=0.312$ & $p=0.044^{b}$ & $p=0.019^{b}$ & $p=0.006^{b}$ \\
\hline \multirow[t]{2}{*}{ Model A } & $0.10 \pm 0.09$ & $0.15 \pm 0.08$ & $0.02 \pm 0.01$ & $0.26 \pm 0.08$ \\
\hline & $p=0.268$ & $p=0.048^{b}$ & $p=0.055$ & $p<0.001^{b}$ \\
\hline \multirow[t]{2}{*}{ Model B } & $0.11 \pm 0.10$ & $0.15 \pm 0.08$ & $0.02 \pm 0.01$ & $0.27 \pm 0.08$ \\
\hline & $p=0.250$ & $p=0.055$ & $p=0.081$ & $p=0.001^{b}$ \\
\hline \multirow[t]{2}{*}{ Model C } & $0.13 \pm 0.10$ & $0.16 \pm 0.08$ & $0.02 \pm 0.01$ & $0.26 \pm 0.08$ \\
\hline & $p=0.180$ & $p=0.050$ & $p=0.096$ & $p=0.002^{b}$ \\
\hline Model D & $0.11 \pm 0.10$ & $0.12 \pm 0.08$ & $0.02 \pm 0.01$ & $0.28 \pm 0.08$ \\
\hline $\begin{array}{l}\text { RBC DHA + EPA } \\
\text { (Q1 vs Q2-4) }\end{array}$ & $p=0.282$ & $p=0.139$ & $p=0.183$ & $p<0.001^{b}$ \\
\hline \multirow[t]{2}{*}{ Model A } & $-0.14 \pm 0.22$ & $-0.45 \pm 0.18$ & $-0.09 \pm 0.03$ & $-0.60 \pm 0.18$ \\
\hline & $p=0.526$ & $p=0.011$ & $p<0.001$ & $p=0.001$ \\
\hline \multirow[t]{2}{*}{ Model B } & $-0.16 \pm 0.22$ & $-0.44 \pm 0.18$ & $-0.09 \pm 0.03$ & $-0.62 \pm 0.19$ \\
\hline & $p=0.462$ & $p=0.016$ & $p=0.001$ & $p<0.001$ \\
\hline \multirow[t]{2}{*}{ Model C } & $-0.17 \pm 0.22$ & $-0.44 \pm 0.18$ & $-0.09 \pm 0.03$ & $-0.61 \pm 0.19$ \\
\hline & $p=0.427$ & $p=0.016$ & $p=0.001$ & $p=0.001$ \\
\hline \multirow[t]{2}{*}{ Model D } & $-0.15 \pm 0.22$ & $-0.34 \pm 0.18$ & $-0.08 \pm 0.03$ & $-0.65 \pm 0.19$ \\
\hline & $p=0.489$ & $p=0.060$ & $p=0.004$ & $p<0.001$ \\
\hline
\end{tabular}

Abbreviations: $\mathrm{Cl}$ = confidence interval; $\mathrm{DHA}$ = docosahexaenoic acid; $\mathrm{EPA}=$ eicosapentaenoic acid; $\mathrm{OR}=$ odds ratio; $\mathrm{RBC}=$ red blood cell.

${ }^{a}$ Model A: Adjusted for age, sex, time interval, and education. Model B: model A with additional adjustment for APOE4 and homocysteine. Model C: model B with additional adjustment for physical activity index and body mass index. Model D: model B with additional adjustment for diabetes mellitus, systolic blood pressure, smoking, atrial fibrillation, prevalent cardiovascular disease, and total cholesterol.

b Significant. lar Health Study, which found that the consumption of fish at least 3 times a week based on dietary questionnaires was associated with a lower risk of subclinical vascular brain abnormalities. ${ }^{24}$ Fatty acids are integral components of biological membranes, and influence membrane fluidity, ion transport, and other functions. ${ }^{25}$ The neuronal cell membrane is no exception; the CNS has the highest concentration of phospholipids in the body. Long-chain omega-3 polyunsaturated fatty acids (PUFA) are abundant in the brain, particularly the omega-3 PUFA DHA and the n-6 PUFA arachidonic acid, both essential fatty acids that are very inefficiently synthesized from shorter-chain dietary precursor $(\alpha$-linolenic acid and linoleic acid, respectively), ${ }^{26}$ and are best obtained preformed from the diet. Measurement of RBC fatty acid composition is a reliable biological indicator of dietary intake of omega-3 PUFAs. A longitudinal study relating RBC PUFA levels with cognitive performance showed that lower concentrations of omega-3 PUFA were associated with a higher risk of cognitive decline in an elderly French cohort followed for 4 years. ${ }^{27}$ Dietary intake of fatty fish is the main source of the omega-3 PUFAs DHA and EPA, and thus although the results of population-based studies are not all in accord, increased fish consumption has been shown by the majority of studies published to date to lower the risk of dementia and cognitive decline. ${ }^{28}$ These findings are particularly interesting when considered along with evidence that biosynthesis of EPA and DHA from their precursor $\alpha$-linolenic acid appears to decrease with age. ${ }^{29} \mathrm{~A}$ recent clinical trial found that daily supplementation with DHA in older adults for 24 weeks improved learning and memory function, ${ }^{30}$ but another trial with DHA supplementation in persons with mild to moderate $\mathrm{AD}$ failed to show attenuation of rates of cognitive and functional decline and brain atrophy. ${ }^{31}$ In a 6-month study ${ }^{32}$ with DHA and EPA in subjects with mild to moderate $\mathrm{AD}$, there was no overall delay the rate of cognitive decline, but a subanalysis in subjects with very mild $\mathrm{AD}$ showed a positive effect, suggesting that the protective effect of omega-3 PUFAs might occur during the earliest stages of cognitive decline.

The biological mechanisms through which omega-3 PUFAs might exert beneficial effects on the brain can be broadly divided into vascular and nonvascular pathways. DHA and EPA exert several favorable effects on the vasculature, including blood pressure reduction, ${ }^{33}$ lowering the risk of thrombosis, ${ }^{34}$ reducing inflammation, ${ }^{35}$ and lowering serum triglyceride levels. ${ }^{36}$ Since vascular risk factors, cerebral atherosclerosis, and stroke have been associated with a higher risk of incident dementia, ${ }^{37}$ omega-3 
PUFAs may delay cognitive and structural brain aging by some combination of these mechanisms. The omega-3 PUFA composition of both lipid rafts and caveolae is known to influence membrane function and the activities of membrane-bound proteins, ${ }^{38}$ suggesting another potential mechanism of action. Other roles of omega-3 PUFAs may be more directly linked to the neurodegenerative pathogenesis of $\mathrm{AD}$, including reduction of amyloid- $\beta$ production, synaptic protection by reducing neuroinflammation and oxidative damage, by increasing levels of brainderived neurotrophic factor, and through reduction of potentially excitotoxic arachidonic acid (omega-6) levels. ${ }^{39}$ Our findings of an association of RBC DHA and EPA levels with total cerebral brain volume and white matter hyperintensity volume but not with a surrogate marker of hippocampal volume, and its association with NP measures linked to vascular cognitive impairment (visual memory, executive function, abstract thinking), suggest that at least in the early preclinical phases, the underlying pathologic mechanisms may be more vascular than neurodegenerative.

The strengths of the current investigation are the focus on middle-aged to elderly subjects who were free of clinical stroke and dementia, the simultaneous availability of both structural and cognitive measures, and the use of a reliable biological measure of omega-3 fatty acid levels. Nonetheless, there are several limitations. Given that the variables used were measured during the most recent examination cycle, there were no subsequent brain MRI and NP data available to measure rates of change in both structural brain volume and cognitive performance measures. In other words, the cross-sectional design of the study precluded examination of the relationship between RBC omega-3 PUFA levels and the development of clinical dementia. In addition, the primarily Caucasian makeup of the Framingham offspring cohort may limit the generalizability of our findings to this racial/ethnic group. The association between lower RBC omega-3 fatty acid levels and markers of accelerated cognitive and structural brain aging observed here should be confirmed in other populations and extended in the future to include dementia outcomes.

\section{AUTHOR CONTRIBUTIONS}

Dr. Tan: study concept/design, analysis/interpretation of data, drafting of manuscript, acquisition of data. Dr. Harris: study concept/design, analysis/interpretation of data, drafting of manuscript, acquisition of data. Dr. Beiser: study concept/design, analysis/interpretation of data, drafting of manuscript, statistical analyses. Dr. Au: analysis/interpretation of data, drafting of manuscript, acquisition of data. J.J. Himali: drafting of manuscript, statistical analyses. Dr. Debette: analysis/interpretation of data, drafting of manuscript. Dr. Pikula: analysis/interpretation of data, drafting of manuscript, acquisition of data. Dr. DeCarli: analysis/interpretation of data, drafting of manuscript, acquisition of data. Dr. Wolf: analysis/interpretation of data, drafting of manuscript, obtaining funding, acquisition of data. Dr. Vasan: analysis/interpretation of data, drafting of manuscript. Dr. Robins: analysis/interpretation of data, drafting of manuscript, acquisition of data. Dr. Seshadri: study concept/design, analysis/ interpretation of data, drafting of manuscript, obtaining funding, acquisition of data.

\section{DISCLOSURE}

Dr. Tan has received research support from the NIH/NIA. Dr. Harris serves on scientific advisory boards for Monsanto, Aker Biomarine, Acasti Pharma, Unilever, Neptune, Omthera, and GlaxoSmithKline; has received funding for travel from Monsanto; has served on the speaker's bureau for GlaxoSmithKline; is employed by Health Diagnostic Laboratory, Inc., which offers blood omega-3 testing; has received research support from Monsanto, Aker Biomarine, Acasti Pharma, Neptune, Omthera, GlaxoSmithKline, and the NIH/NHLBI; and is the owner of OmegaQuant, LLC, a company that offers blood fatty acid testing. Dr. Beiser receives publishing royalties for Introductory Applied Statistics (Brooks Cole, 2005) and receives research support from the NIH (NINDS, NIA, NHLBI). Dr. Au receives research support from the NIH (NIA, NINDS). J.J. Himali, Dr. Debette, and Dr. Pikula report no disclosures. Dr. DeCarli serves as Editor-in-Chief for Alzheimer Disease and Associated Disorders; serves as a consultant for Avid Radiopharmaceuticals, Inc. and Bayer Schering Pharma; and receives research support from the NIH (NIA, NHLBI). Dr. Wolf receives publishing royalties from the 5th edition of Stroke: Pathophysiology, Diagnosis, and Management (Elsevier, 2008) and receives research support from the NIH (NHLBI, NINDS, NIA). Dr. Vasan reports no disclosures. Dr. Robins receives research support from the NIH/NHLBI. Dr. Seshadri serves as an Associate Editor for the Journal of Alzheimer's Disease and on the editorial boards of Stroke and Neurology ${ }^{\circledR}$; and receives research support from the NIH (NIA, NINDS, NHLBI).

Received June 16, 2011. Accepted in final form October 26, 2011.

\section{REFERENCES}

1. He K, Song Y, Daviglus ML, et al. Accumulated evidence on fish consumption and coronary heart disease mortality: a meta-analysis of cohort studies. Circulation 2004;109: 2705-2711.

2. Daviglus ML, Stamler J, Orencia AJ, et al. Fish consumption and the 30-year risk of fatal myocardial infarction. N Engl J Med 1997;336:1046-1053.

3. He K, Rimm EB, Merchant A, et al. Fish consumption and risk of stroke in men. JAMA 2002;288:3130-3136.

4. Kalmijn S, Launer LJ, Ott A, Witteman JC, Hofman A, Breteler MM. Dietary fat intake and the risk of incident dementia in the Rotterdam Study. Ann Neurol 1997;42: $776-782$

5. Morris MC, Evans DA, Bienias JL, et al. Consumption of fish and n-3 fatty acids and risk of incident Alzheimer disease. Arch Neurol 2003;60:940-946.

6. Laurin D, Verreault R, Lindsay J, Dewailly E, Holub BJ. Omega-3 fatty acids and risk of cognitive impairment and dementia. J Alzheimers Dis 2003;5:315-322.

7. Hjartaker A, Lund E, Bjerve KS. Serum phospholipid fatty acid composition and habitual intake of marine foods registered by a semi-quantitative food frequency questionnaire. Eur J Clin Nutr 1997;51:736-742.

8. Philibert A, Vanier C, Abdelouahab N, Chan HM, Mergler D. Fish intake and serum fatty acid profiles from freshwater fish. Am J Clin Nutr 2006;84:1299-1307.

9. Schaefer EJ, Bongard V, Beiser AS, et al. Plasma phosphatidylcholine docosahexaenoic acid content and risk of dementia and Alzheimer disease: the Framingham Heart Study. Arch Neurol 2006;63:1545-1550.

10. Arab L. Biomarkers of fat and fatty acid intake. J Nutr 2003;133(suppl 3):925S-932S. 
11. Harris WS, Thomas RM. Biological variability of blood omega-3 biomarkers. Clin Biochem 2010;43:338-340.

12. Harris WS, Sands SA, Windsor SL, et al. Omega-3 fatty acids in cardiac biopsies from heart transplantation patients: correlation with erythrocytes and response to supplementation. Circulation 2004;110:1645-1649.

13. Seshadri S, Wolf PA, Beiser A, et al. Stroke risk profile, brain volume, and cognitive function: the Framingham Offspring Study. Neurology 2004;63:1591-1599.

14. DeCarli C, Massaro J, Harvey D, et al. Measures of brain morphology and infarction in the Framingham Heart Study: establishing what is normal. Neurobiol Aging 2005; 26:491-510.

15. Jeerakathil T, Wolf PA, Beiser A, et al. Stroke risk profile predicts white matter hyperintensity volume: the Framingham Study. Stroke 2004;35:1857-1861.

16. DeCarli C, Murphy DG, McIntosh AR, Teichberg D, Schapiro MB, Horwitz B. Discriminant analysis of MRI measures as a method to determine the presence of dementia of the Alzheimer type. Psychiatry Res 1995;57:119130 .

17. Au R, Seshadri S, Wolf PA, et al. New norms for a new generation: cognitive performance in the Framingham Offspring Cohort. Exp Aging Res 2004;30:333-358.

18. Elias MF, Beiser A, Wolf PA, Au R, White RF, D'Agostino RB. The preclinical phase of Alzheimer disease: a 22-year prospective study of the Framingham Cohort. Arch Neurol 2000;57:808-813.

19. Hachinski V, Iadecola C, Petersen RC, et al. National Institute of Neurological Disorders and Stroke-Canadian Stroke Network vascular cognitive impairment harmonization standards. Stroke 2006;37:2220-2241.

20. Harris WS, DiRienzo MA, Sands SA, George C, Jones PG, Eapen AK. Stearidonic acid increases the red blood cell and heart eicosapentaenoic acid content in dogs. Lipids 2007; 42:325-333.

21. Aarsetoey H, Aarsetoey R, Lindner T, Staines H, Harris WS, Nilsen DW. Low levels of the omega-3 index are associated with sudden cardiac arrest and remain stable in survivors in the subacute phase. Lipids 2011;46:151-161.

22. Kannel WB, Belanger A, D’Agostino R, Israel I. Physical activity and physical demand on the job and risk of cardiovascular disease and death: the Framingham Study. Am Heart J 1986;112:820-825.

23. Harris WS, Poston WC, Haddock CK. Tissue n-3 and n-6 fatty acids and risk for coronary heart disease events. Atherosclerosis 2007;193:1-10.

24. Virtanen JK, Siscovick DS, Longstreth WT Jr, Kuller LH, Mozaffarian D. Fish consumption and risk of subclinical brain abnormalities on MRI in older adults. Neurology 2008;71:439-446.

25. Florent-Bechard S, Malaplate-Armand C, Koziel V, et al. Towards a nutritional approach for prevention of Alzhei- mer's disease: biochemical and cellular aspects. J Neurol Sci 2007;262:27-36.

26. Brenna JT. Efficiency of conversion of alpha-linolenic acid to long chain n-3 fatty acids in man. Curr Opin Clin Nutr Metab Care 2002;5:127-132.

27. Heude B, Ducimetiere P, Berr C. Cognitive decline and fatty acid composition of erythrocyte membranes: the EVA Study. Am J Clin Nutr 2003;77:803-808.

28. Cole GM, Ma QL, Frautschy SA. Omega-3 fatty acids and dementia. Prostaglandins Leukot Essent Fatty Acids 2009; 81:213-221.

29. Muskiet FA, Fokkema MR, Schaafsma A, Boersma ER, Crawford MA. Is docosahexaenoic acid (DHA) essential? Lessons from DHA status regulation, our ancient diet, epidemiology and randomized controlled trials. J Nutr 2004; 134:183-186.

30. Yurko-Mauro K, McCarthy D, Rom D, et al. Beneficial effects of docosahexaenoic acid on cognition in age-related cognitive decline. Alzheimers Dement 2010;6:456-464.

31. Quinn JF, Raman R, Thomas RG, et al. Docosahexaenoic acid supplementation and cognitive decline in Alzheimer disease: a randomized trial. JAMA 2010;304:1903-1911.

32. Freund-Levi Y, Eriksdotter-Jonhagen M, Cederholm T, et al. Omega-3 fatty acid treatment in 174 patients with mild to moderate Alzheimer disease: OmegAD study: a randomized double-blind trial. Arch Neurol 2006;63:14021408.

33. Bonaa KH, Bjerve KS, Straume B, Gram IT, Thelle D. Effect of eicosapentaenoic and docosahexaenoic acids on blood pressure in hypertension: a population-based intervention trial from the Tromsø study. N Engl J Med 1990; 322:795-801

34. Keli SO, Feskens EJ, Kromhout D. Fish consumption and risk of stroke: The Zutphen Study. Stroke 1994;25:328332.

35. Calder PC. n-3 Fatty acids and cardiovascular disease: evidence explained and mechanisms explored. Clin Sci 2004; 107:1-11.

36. Kelley DS, Siegel D, Vemuri M, Chung GH, Mackey BE. Docosahexaenoic acid supplementation decreases remnant-like particle-cholesterol and increases the (n-3) index in hypertriglyceridemic men. J Nutr 2008;138: $30-35$.

37. Viswanathan A, Rocca WA, Tzourio C. Vascular risk factors and dementia: how to move forward? Neurology 2009;72:368-374.

38. Ma DW, Seo J, Switzer KC, et al. n-3 PUFA and membrane microdomains: a new frontier in bioactive lipid research. J Nutr Biochem 2004;15:700-706.

39. Cole GM, Frautschy SA. DHA may prevent age-related dementia. J Nutr 2010;140:869-874. 